\title{
ESTRUTURA POPULACIONAL DE HYALE MEDIA (DANA) \\ (AMPHIPODA, GAMMARIDEA, HYALIDAE), HABITANTE DOS FITAIS DE CAIOBÁ, MATINHOS, PARANÁ, BRASIL
}

\author{
Janete Dubiaski-Silva ${ }^{2}$ \\ Setuko Masunari ${ }^{3}$
}

\begin{abstract}
PopUlation STRUCTURE OF THE SEAWEED DWELLER HYale mEdIA (DANA) (Amphipoda, Gammaridea, Hyalidae) from Caiobá, Matinhos, Paraná, Brazil. A study of correlation between the total body length and the somites length was carried out in a population of Hyale media (Dana, 1857), in order to know which somite or group of somites has the highest correlation index with the total body length. As the sum of the length of the first to fourth pereonites showed the highest linear correlation index $(Y=0.0764+0.2736 \mathrm{X} ; \mathrm{r}=0.9723)$, this meristic parameter was chosen to describe the population structure of the species. The following aspects were treated: distribution of the body size classes in the various phytals, population composition, seasonal fluctuation of population density, relative frequency of the ovigerous females and correlation between the body length and the number of eggs inside the marsupium of the ovigerous females. The amphipods were obtained from the seasonal collections of six phytals from a rocky seashore of Caiobá, Paraná State: Pterosiphonia pennata (Roth) Falkenberg, Gymnogongrus griffithsiae (Turner) Martius, Pterocladia capillacea (Gmelin) Bornet \& Thured, Sargassum cymosum Garth, Gelidium sp and Ulva fasciata Delile; they did not occurred in Padina gymnospora (Kútsing) Vickers and Porphyra atropurpurea (Olivi) De Toni. The air temperature oscillated from $16^{\circ} \mathrm{C}$ (winter and autumn) to $23^{\circ} \mathrm{C}$ (summer), the surface water temperature from $17^{\circ} \mathrm{C}$ (winter) to $25^{\circ} \mathrm{C}$ (summer) and the surface water salinity, from $29.3 \%$ (autumn) to $32.8 \%$ (winter). The density of Hyale media varied from 0.20 ind. $^{-1}$ (in Ulva) to 26.37 ind. $\mathrm{g}^{-1}$ (in Pterosiphonia) of alga-substratum weigth, and the population was distributed mainly in branched algae. It was determined three size classes in the population, within a range from 0.01 to $2.99 \mathrm{~mm}$ of pereonits $1-4$ length. Small amphipods prefer finely branched algae like Gymnogongrus and Pterosiphonia, whereas broad-thallii or less branched algae such as Sargassum, Pterocladia, Gelidium and Ulva harbour proporcionally high number of large individuals. The life cycle of Hyale media takes place wholly in the phytals and the species reproduces continually all year round: males, ovigerous females and juveniles are present every season. The highest female reproductive activity occurs in winter and the juveniles are more numerous in summer. The number of eggs inside the marsupium and the pereonites 1-4 length has a linear correlation ( $\mathrm{Y}=-9,9682+12,0729 \mathrm{X} ; \mathrm{r}=0.8024)$.

KEY WORDS. Hyale media, population, phytal, correlation, density, Matinhos, Brazil
\end{abstract}

1) Contribuição número 962 do Departamento de Zoologia, Universidade Federal do Paraná.

2) Departamento de Ciências Naturais, Centro Universitário de Três Lagoas, Universidade Federal do Mato Grosso do Sul. Caixa Postal 210, 79603-011 Três Lagoas, Mato Grosso do Sul, Brasil.

3) Departamento de Zoologia, Universidade Federal do Paraná. Caixa Postal 19020, 81531-990 Curitiba, Paraná, Brasil.

Revta bras. Zool. 15 (1): 59 - 71, 1998 
O fital constitui um dos ambientes menos conhecidos do litoral paranaense, havendo poucos estudos sobre ele (JAKOBI 1954, 1962; LOYOLA E SILVA 1960; DUTRA 1988; MASUNARI 1986 e DUBIASKI-SILVA \& MASUNARI 1995), apesar de largamente conhecido no exterior (ver revisão em MASUNARI \& FORNERIS 1981) após a proposição do termo por REMANE (1933).

Neste ecossistema, Amphipoda e Mollusca são os grupos dominantes da macrofauna na maioria dos estudos já realizados (MASUNARI 1987). Sendo os Amphipoda um grupo numericamente expressivo no fital, a sua participação na cadeia trófica das comunidades marinhas costeiras foi diretamente relacionada à produção pesqueira (KIKUCHI \& PÉRÈS 1977).

Estudos experimentais de Amphipoda de fitais foram realizados por vários autores focalizando aspectos como preferências alimentares (BRAWLEY \& FEY 1987; DUFFY 1990; VIEJO \& ARRONTES 1992) e influência da complexidade estrutural do habitat na defesa contra os predadores (RYER 1988; HACKER \& STENECK 1990; SCHNEIDER \& MANN 1991b).

A predação de Amphipoda de fitais e de gramas marinhas por peixes foi descrita por RYER \& ORTH (1987), LUCZKOVICH (1988) e GIBBONS (1988), enquanto a composição de espécies e abundância relativa por, entre outros, LEWIS (1987), HACKER \& STENECK (1990), SchnEIDER \& MANN (1991a) e Dubiaski-Silva \& MASUNARI (1995).

Aspectos populacionais de Hyale media (Dana, 1857) foram tratados por WAKABARA et al. (1983), TARARAM et al. (1986) e DUBIASKI-SILVA \& MASUNARI (1995); de hábitos alimentares e assimilação de alimento por TARARAM et al. (1985, 1990), de crescimento e reprodução por LEITE (1996a), de fecundidade por LEITE (1996b) e a morfologia dos oostegitos das fêmeas por LEITE et al. (1986). LANCELLOTTI \& TRUCCO (1993) estudaram os padrões de distribuição e coexistência desta espécie com cinco outras, pertencentes ao mesmo gênero, em Coquimbo, Chile, e sua associação com Cellana tramoserica (Sowerby) foi investigada experimentalmente por UNDERWOOD \& VERTEGEN (1988).

Com a finalidade de descrever a estrutura populacional de Hyale media, os seguintes aspectos são tratados no presente trabalho: 1) Escolha do melhor parâmetro merístico para inferir o comprimento do corpo da espécie; 2) distribuição de densidade destes animais nos diversos fitais, discriminando o estágio de desenvolvimento e o sexo; 3) flutuação estacional de densidade da população; 4) relações entre a espécie e os parâmetros abióticos e bióticos estudados; 5) flutuação estacional e frequência relativa das fêmeas ovígeras; 6) correlação entre o tamanho do corpo das fêmeas ovígeras e o número de ovos presentes no respectivo marsúpio.

\section{MATERIAL E MÉTODOS}

O material biológico é proveniente dos fitais do litoral de matacões e costões

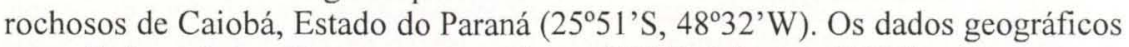
e geológicos da região constam em JOLY (1951), MAACK (1968) e BIGARELLA (1978). O mapa do local de estudo e a descrição da metodologia de coleta encontram-se em DUBIASKI-SILVA \& MASUNARI (1995). Entretanto, algumas informações adicionais ou relevantes são apresentadas no presente trabalho. 
As coletas sazonais dos fitais foram realizadas durante a baixamar nas seguintes datas: 06/agosto/86 (inverno), $1 \%$ novembro/86 (primavera), $15 /$ março/87 (verão) e $28 / \mathrm{maio} / 87$ (outono). A temperatura do ar variou de $16^{\circ} \mathrm{C}$ (inverno e outono) a $23^{\circ} \mathrm{C}$ (verão), a da água de superfície de $17^{\circ} \mathrm{C}$ (inverno) a $25^{\circ} \mathrm{C}$ (verão) e a salinidade de $29,3 \%$ o (outono) a 32,8\% (inverno). A altura da maré mínima variou de $0,0 \mathrm{~m}$ (inverno, primavera e outono) a $0,2 \mathrm{~m}$ (verão) nos dias de coleta. $\mathrm{O}$ número de dias com altura da maré prevista inferior a $0,0 \mathrm{~m}$ foi de 13 para o mês de agosto/ 86 , 17 para novembro/86, 9 para março/87 e 7 para maio/87 (DHN 1986 e 1987). No outono, embora a previsão da altura da maré registrasse $0,0 \mathrm{~m}$, visivelmente a maré não abaixou até esta altura, fato relacionado ao tempo chuvoso. Novembro foi o mês com maior número de dias com marés extremas e, portanto, o mês de maior tempo de exposição à dessecação na zona entremarés (DUBIASKI-SILVA \& MASUNARI 1995).

Os exemplares de Hyale media ocorreram em seis dos oito fitais estudados: Pterosiphonia pennata (Roth) Falkenberg, Gymnogongrus griffithsiae (Turner) Martius, Pterocladia capillacea (Gmelin) Bornet \& Thuret, Sargassum cymosum Garth, Gelidium sp. e Ulva fasciata Delile; não foram registrados em Padina gymnospora (Kützing) Vickers e Porphyra atropurpurea (Olivi) De Toni. Os fitais estão referidos pelo nome do gênero das algas no presente trabalho.

A diferenciação sexual de $H$. media foi feita com base nos caracteres sexuais primários (presença de pênis ou oostegitos) e/ou secundário (tamanho do segundo gnatópodo). Aqueles indivíduos que ainda não apresentavam tais caracteres evidentes, foram considerados juvenis. As fêmeas maturas foram separadas em ovigeras (com o marsúpio contendo ovos ou embriões) e não ovígeras (com oostegitos, porém, sem marsúpio). Na contagem de ovos, o marsúpio foi rompido com um estilete.

Foi realizado um estudo da melhor variável morfológica para inferir o comprimento do corpo de $H$. media devido à dificuldade em se medir o comprimento do corpo destes animais, que se curvam quando fixados. Para tanto, foram separados 130 indivíduos, compostos por machos, fêmeas e juvenis em proporções aproximadas, dos quais foram tomadas medidas do comprimento da cabeça, de todos os somitos do corpo isoladamente e do corpo inteiro (da extremidade anterior do rostro até o final do último urossomito). Após, a correlação foi feita entre as variáveis a seguir e o comprimento total do corpo: comprimento da cabeça, soma dos pereonitos 1, 2 e 3, soma dos pereonitos 1 a 4, pereonitos 4, 5, 6 e 7 isoladamente, pleonitos 1 , 2 e 3 isoladamente, soma dos urossomitos 1,2 e 3 e urossomitos 1,2 e 3 isoladamente. Com estes dados, foi determinada a parte do corpo que apresentou a correlação mais forte com a variação do comprimento total do corpo. Estabelecida a equação de regressão para os referidos 130 indivíduos, esta foi utilizada no tratamento merístico para toda a população de $H$. media, que teve os indivíduos agrupados em três classes de comprimento dos pereonitos 1-4: $<1,0 \mathrm{~mm}$, entre 1,0 e $2,0 \mathrm{~mm}$ e $>2,0 \mathrm{~mm}$, as quais foram tratadas como classes de tamanho I, II e III, respectivamente. A amplitude destas três classes foi estabelecida em função do tamanho dos juvenis $(0,61-0,82 \mathrm{~mm})$ e dos adultos (machos $0,89-2,3 \mathrm{~mm}$ e fêmeas $0,84-2,72 \mathrm{~mm}$ ). Assim à classe de tamanho I estão contidos todos os juvenis, a 
maioria dos adultos pertence à classe de tamanho II e os adultos maiores e raros, à classe III.

Para se verificar se havia diferenças significativas do tamanho dos Gammaridea entre as algas estudadas, foi utilizada a ANOVA de Friedman, executada pela versão 4.2 do programa Statistica para Windows.

\section{RESULTADOS}

Foram coletados 4511 Amphipoda Gammaridea pertencentes a sete espécies durante o período de coleta: Ampithoe ramondi Audouin, 1816, Cymadusa filosa Savigny, 1852, Elasmopus pectenicrus Bate, 1857, Hyale media Dana, 1857, Hyale sp 1, Jassa falcata Montagu, 1895 e Sunampithoe pelagica H. Milne-Edwards, 1830. Destas, Hyale media foi a mais abundante, totalizando 1570 indivíduos ou 34,28\% dos Gammaridea estudados; ocorreu em todas as estações do ano estudadas e a densidade variou de 0,20 ind. $\mathrm{g}^{-1}$ em Ulva a 26,37 ind.g ${ }^{-1}$ em Pterosiphonia (DUBIASKI-SILVA \& MASUNARI 1995).

O coeficiente médio de adsorção das algas-substrato (segundo WIESER 1951) variaram de $16,87 \%$ em Gelidium a 63,04\% em Pterosiphonia. A quantidade média de sedimento retido nos talos da alga-substrato (DAHL 1948) variou de 46,89 mg. $\mathrm{g}^{-1}$ em Gelidium a 2254,06 mg. $\mathrm{g}^{-1}$ em Gymnogongrus e, a distância média entre as ramificações (somente para as algas-substrato ramificadas), de $1,16 \mathrm{~mm}$ para Gymnogongrus a 8,95mm para Sargassum (DUBIASKI-SILVA \& MASUNARI 1995).

\section{Correlação entre o comprimento total do corpo e o comprimento dos somitos do corpo}

Dentre as correlações estudadas entre o comprimento total do corpo e os vários somitos ou conjunto de somitos (Tab. I), a mais forte foi com a da soma dos quatro primeiros pereonitos, cujo coeficiente de correlação teve o valor $\mathrm{r}=0,9723$ $(p<0.05)$ e a equação de regressão encontrada foi: $Y=0,0764+0,2736 \mathrm{X}$ (Fig. 1)

\section{Distribuição de densidade das classes de tamanho do corpo nos fitais}

A figura 2 mostra a distribuição de densidade das classes de tamanho do corpo (comprimento dos pereonitos 1-4) de $H$. media nos diversos fitais nas amostras de inverno, primavera, verão e outono.

As três classes de tamanho ocorreram nas quatro estações do ano. Entretanto, a classe de tamanho I (constituída predominantemente por juvenis, embora machos e fêmeas também estejam presentes) foi a mais abundante e mais frequente em todas as estações do ano, indicando um recrutamento contínuo de novos indivíduos na população durante $o$ ano. Esta classe de tamanho atingiu o máximo de densidade no verão com 44,93 ind. ${ }^{-1}$ em Gymnogongrus, seguido de Pterosiphonia com 30,76 ind. $\mathrm{g}^{-1}$; na primavera, ela foi abundante somente em Pterosiphonia com 33,84 ind. $g^{-1}$.

A classe de tamanho II (constituída por machos e fêmeas), menos abundante do que a I, mostrou densidades que oscilaram de 0,06 ind.g ${ }^{-1}$ em Sargassum (outono) a 20,67 ind.g ${ }^{-1}$ em Gymnogongrus (verão). 


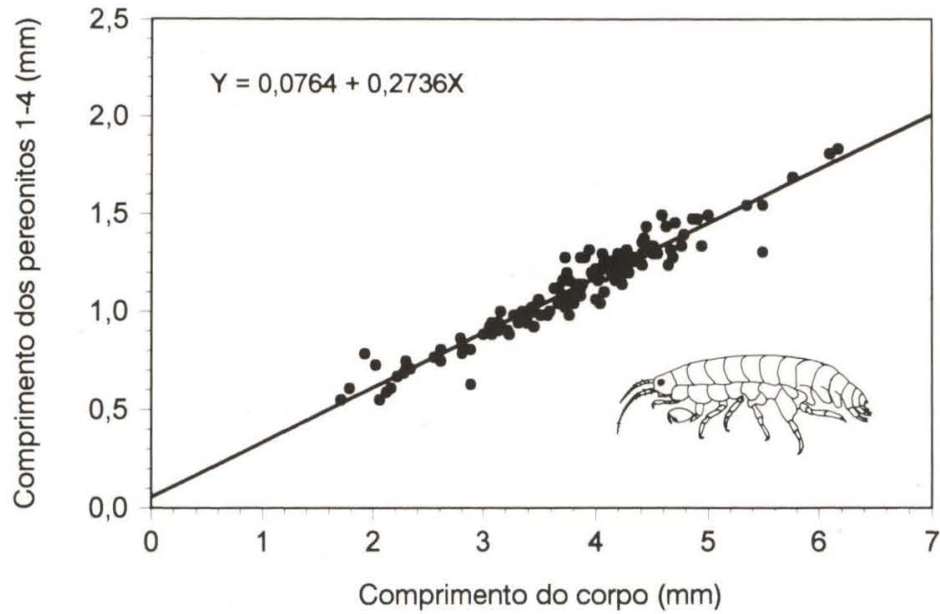

Fig. 1. Hyale media. Regressão do comprimento total do corpo $(\mathrm{mm})$ para o comprimento dos pereonitos 1-4 $(\mathrm{mm})$. Equação da reta $Y=0,0764+0,2736 X ; r=0,9723$.

Tabela I. Hyale media. Matriz de correlação entre o comprimento da cabeça (C), soma dos pereonitos 1,2 e 3 ( $\operatorname{Pr} 123$ ), pereonito 4 (Pr4), soma dos pereonitos 1,2,3 e 4 ( $\operatorname{Pr} 1234$ ), pereonito $5(\operatorname{Pr} 5)$, pereonito 6 ( $\mathrm{Pr} 6)$, pereonito $7(\operatorname{Pr} 7)$, pleonito 1 (PI1), pleonito $2(\mathrm{PI} 2)$, pleonito $3(\mathrm{PI} 3)$, urossomitos 1,2 e 3 (Ur123) e o comprimento total do corpo (Total).

\begin{tabular}{|c|c|c|c|c|c|c|c|c|c|c|c|c|}
\hline & C & $\operatorname{Pr} 123$ & $\operatorname{Pr} 4$ & Pr1234 & Pr5 & Pr6 & $\mathrm{Pr} 7$ & PI1 & $\mathrm{P} 12$ & PI3 & Ur123 & Total \\
\hline $\bar{c}$ & 1,0000 & & & & & & & & & & & \\
\hline Pr123 & 0,8966 & 1,0000 & & & & & & & & & & \\
\hline Pr4 & 0,8541 & 0,8737 & 1,0000 & & & & & & & & & \\
\hline $\operatorname{Pr} 1234$ & 0.9080 & 0,9886 & 0,9358 & 1,0000 & & & & & & & & \\
\hline Pr5 & 0,8845 & 0,8961 & 0,9180 & 0,9267 & 1,000 & & & & & & & \\
\hline Pr6 & 0,8676 & 0,9115 & 0,8650 & 0,9219 & 0,9073 & 1,0000 & & & & & & \\
\hline $\operatorname{Pr} 7$ & 0,8934 & 0,8992 & 0,8643 & 0,9149 & 0,9034 & 0,9031 & 1,0000 & & & & & \\
\hline $\mathrm{Pl1}$ & 0,8908 & 0,8849 & 0,8807 & 0,9069 & 0,9041 & 0,8923 & 0,9444 & 1,0000 & & & & \\
\hline $\mathrm{PI} 2$ & 0,8905 & 0,8624 & 0,8780 & 0,8915 & 0,8964 & 0,8669 & 0,9247 & 0,9405 & 1,0000 & & & \\
\hline $\mathrm{PI} 3$ & 0,8652 & 0,8416 & 0,8245 & 0,8617 & 0,8679 & 0,8605 & 0,9152 & 0,9015 & 0,9379 & 1,0000 & & \\
\hline Ur123 & 0,8348 & 0,8718 & 0,7966 & 0,8746 & 0,8396 & 0,8601 & 0,8905 & 0,8837 & 0,8958 & 0,9109 & 1,0000 & \\
\hline Total & 0,9382 & 0,9566 & 0,9212 & 0.9723 & 0,9502 & 0,9468 & 0,9627 & 0,9599 & 0,9540 & 0,9365 & 0,9289 & 1,0000 \\
\hline
\end{tabular}

A classe de tamanho III foi constituída, praticamente, apenas por machos, pois apenas duas fêmeas ovígeras se enquadraram nesta categoria. Mostrou ser a classe de tamanho menos numerosa, atingindo o máximo de densidade de 0,71 ind. $g^{-1}$ em Pterocladia, no inverno.

De uma forma geral, indivíduos de menor tamanho foram mais frequentes e mais abundantes na primavera e no verão, ao passo que, indivíduos maiores mostraram densidades mais altas no inverno e no outono (Fig. 2).

Indivíduos da classe de tamanho I não foram registrados em Ulva. O teste de Friedman revelou que esta classe ocorreu em densidades significativamente maiores em Pterosiphonia e Gymnogongrus, quando comparadas com as de Gelidium. As densidades registradas em Sargassum e Pterocladia não diferiram significativamente das três algas-substrato anteriormente citadas, nem entre si $\left(\mathrm{X}^{2}=\right.$ 14,800 , G.L. $=4$, PROB. $=5,135 \cdot 10^{-3}$, D.M.S. $\left.=12,20\right)$. 

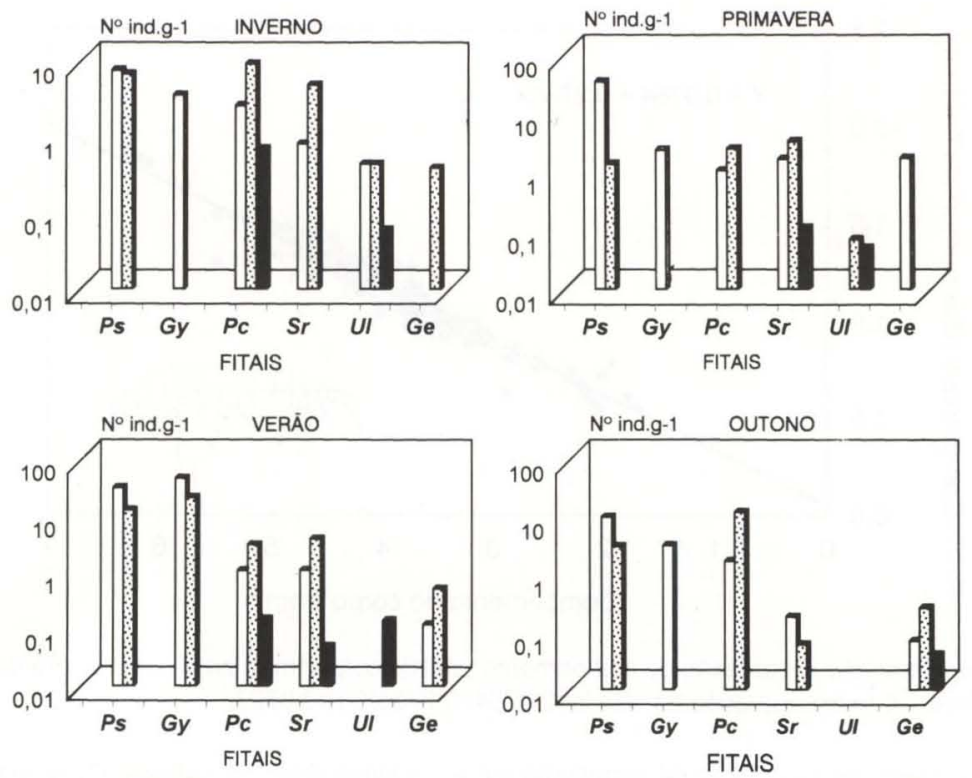

$\square$ Classe I $:$ Classe II $\square$ Classe III

Fig. 2. Hyale media. Distribuição de densidade $\left(n^{\circ}\right.$ ind. $\left.\mathrm{g}^{-1}\right)$ das classes de comprimento dos pereonitos 1-4 nos diversos fitais nas amostras de inverno/86, primavera/86, verão/87 e outono/87. Tamanho I=< $1,0 \mathrm{~mm}$; tamanho $\|=$ entre 1,0 e $2,0 \mathrm{~mm}$ e tamanho $\| I=>2,0 \mathrm{~mm}$. A escala (logarítmica) de densidade é diferente para cada estação do ano. (Ps) Pterosiphonia, (Gy) Gymnogongrus, (Pc) Pterocladia, (Sr) Sargassum, (UI) Ulva, (Ge) Gelidium.

As densidades de $H$. media da classe II não apresentaram diferenças significativas entre todas as espécies de alga-substrato $\left(\mathrm{X}^{2}=5,4\right.$, G.L. $=4$, PROB. $=$ 0,2487). Finalmente, indivíduos da classe III não ocorreram em Pterosiphonia e Gymnogongrus e não diferiram significativamente dentre as demais algas-substrato $\left(\mathrm{X}^{2}=9,050\right.$, G.L. $=4$, PROB. $\left.=0,0599\right)$.

Os dados indicam uma tendência das menores classes de tamanho de $H$. media serem encontradas preferencialmente em algas mais finamente ramificadas como Gymnogongrus e Pterosiphonia. As algas com talos menos ramificados ou foliáceos como Sargassum, Pterocladia, Gelidium e Ulva abrigaram proporcionalmente maior número de indivíduos das classes de maior tamanho.

\section{Composição da população}

A figura 3 mostra a distribuição de densidade de machos, fêmeas e juvenis de H. media nas diversas algas-substrato, nas quatro estações do ano.

De um modo geral, algas foliáceas como Ulva e pouco ramificadas como Sargassum e Gelidium abrigaram, proporcionalmente, maiores densidades de adultos de $H$. media do que juvenis. Por outro lado, Pterocladia, Pterosiphonia e Gymnogongrus, de talos mais ramificados, mostraram altas densidades da espécie, cuja maior contribuição foi a de juvenis, na maioria das amostras. 


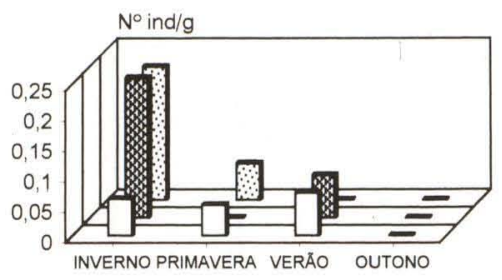

Uha

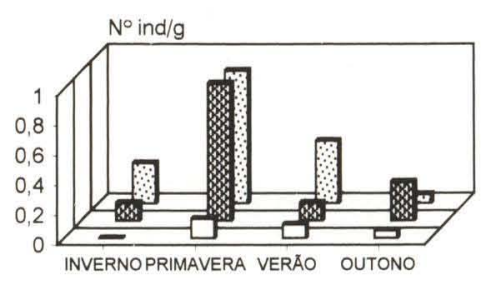

Gelidium

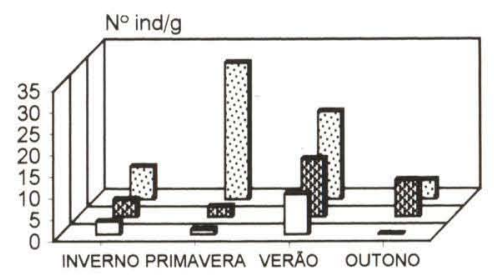

Pterosiphonia

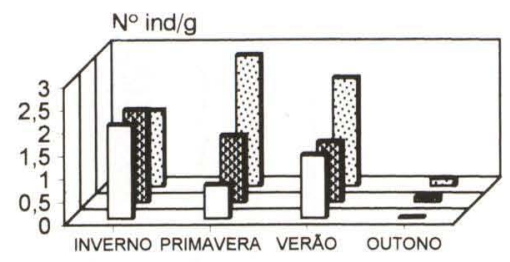

Sargassum

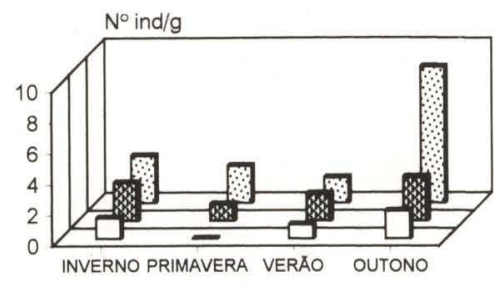

Pteracladia

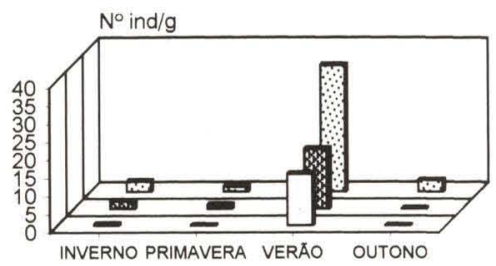

Gymnogongrus

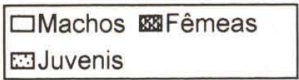

Fig. 3. Hyale media. Distribuição de densidade $\left(n^{0}\right.$ ind $\left.\mathrm{g}^{-1}\right)$ de machos, fêmeas e juvenis nos diversos fitais, nas amostras de inverno/86, primavera/86, verão/87 e outono/87. A escala de densidade é diferente para cada fital.

Juvenis de $H$. media dominaram em Pterosiphonia no inverno e na primavera, Gymnogongrus no verão e Pterocladia no outono (Fig. 3). Esta distribuição diferenciada de acordo com as estações do ano pode estar relacionada com a frequência de marés baixas extremas em cada época do ano. No inverno e na primavera, quando a exposição à dessecação é mais prolongada, os juvenis de $H$. media povoaram Pterosiphonia que se localiza nos níveis inferiores da zona entremarés. Já no verão, quando a oscilação do nível da maré não é tão acentuada, os juvenis desta espécie colonizaram Gymnogongrus que, em geral, habita locais superiores. Pterocladia ocorrendo em faixas intermediárias, abrigou um valor intermediário de densidade. Também, a ramificação menos intensa desta alga-substrato poderia explicar estes valores.

Dentre os adultos, a proporção de sexos foi de 1:1 no inverno (253 machos e 283 fềmeas), 1:3 na primavera ( 73 e 223), $1: 2$ no verão $(230$ e 440$)$ e 1:4 no outono (24 e 98). 

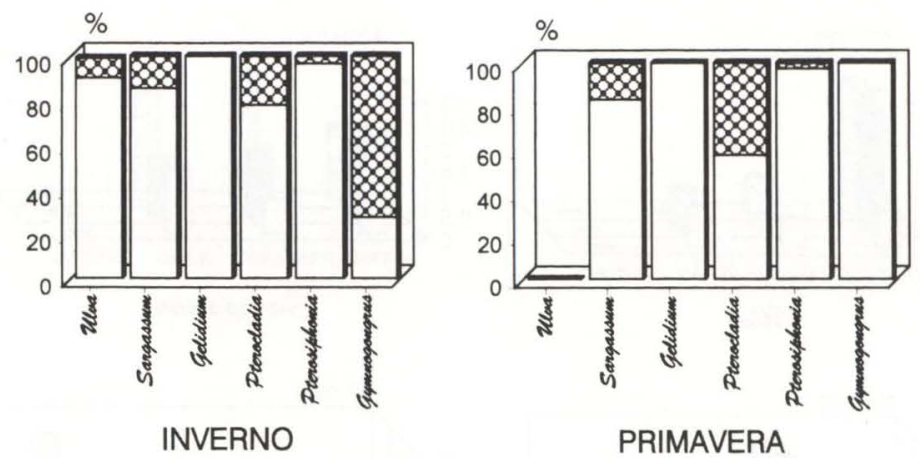

PRIMAVERA
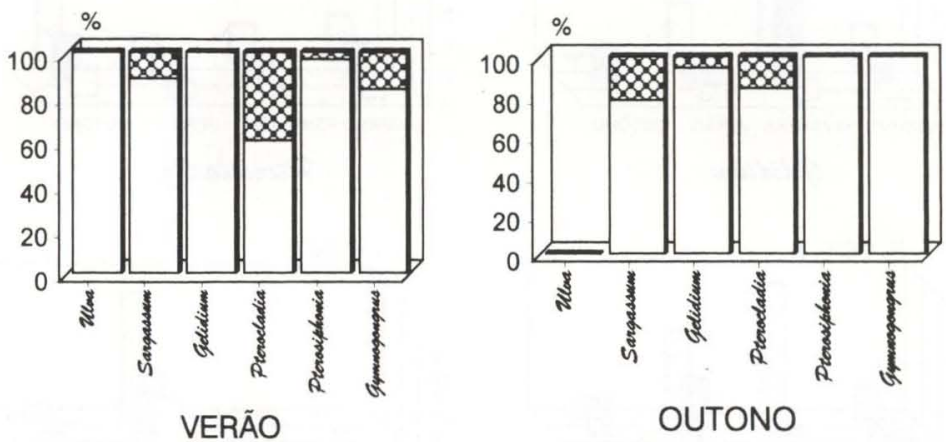

$\square$ Năo ovigeras DOvigeras

Fig. 4. Hyale media. Proporção relativa (\%) de fêmeas ovigeras e não ovígeras nas amostras de inverno/86, primavera/86, verão/87 e outono/87.

\section{Flutuação sazonal e frequência relativa das fêmeas ovígeras}

Fêmeas ovígeras de $H$. media foram registradas em todas as estações do ano indicando uma reprodução contínua da espécie durante o ano. Entretanto, a maior atividade reprodutiva das fêmeas foi registrada no inverno $(21,53 \%$ do total de fêmeas), seguindo-se o outono $(12,61 \%)$, verão $(11,16 \%)$ e primavera $(10,83 \%)$ (Fig. 4). Aparentemente, este contingente de fêmeas ovígeras registradas no inverno e outono contribuiu para o recrutamento de juvenis registrados no verão e primavera, especialmente em Pterosiphonia e em Gymnogongrus (Fig. 3).

\section{Correlação entre o comprimento dos pereonitos 1-4 e o número de ovos no marsúpio das fêmeas ovígeras}

A figura 5 mostra a correlação entre o comprimento dos quatro primeiros pereonitos das fêmeas ovígeras de $H$. media e o número de ovos ou embriões contidos no interior do respectivo marsúpio. O número de ovos ou embriões no marsúpio variou de 2 a 28 . Estes valores-limite ocorreram em fêmeas com $0,96 \mathrm{~mm}$ e $2,72 \mathrm{~mm}$ de comprimento dos pereonitos $1-4$, respectivamente, as quais foram, também, a menor e a maior fêmeas de $H$. media registradas no presente estudo. 
Esta correlação apresentou uma tendência linear, com $r=0,8024(p<0,05)$ e a equação de regressão encontrada foi: $Y=-9,9682+12,0729 X$.

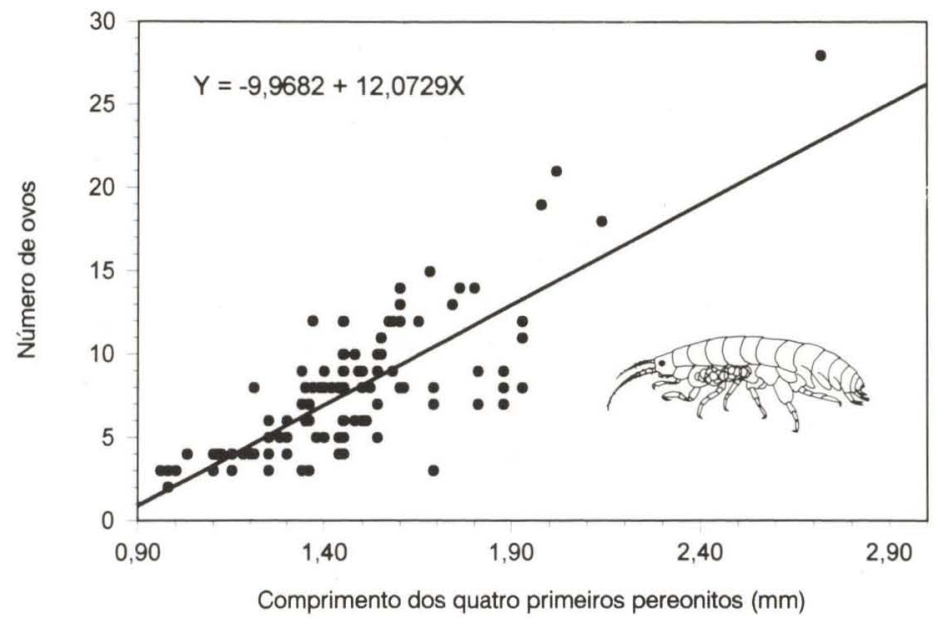

Fig. 5. Hyale media. Regressão do comprimento dos pereonitos 1-4 $(\mathrm{mm})$ para o número de ovos das fêmeas ovigeras. Equação da reta: $Y=-9,9682+12,0729 X ; r=0,8024$.

\section{DISCUSSÃO}

EDGAR (1983a, 1983b) e HACKER \& STENECK (1990) encontraram densidades de diferentes classes de tamanho de Gammaridea distribuindo-se de maneira diversa em várias espécies de algas. O primeiro autor atribuiu o fato à largura das ramificações das algas, encontrando densidades de indivíduos de menores tamanhos significativamente maiores em algas ramificadas. Já os últimos, atribuíram seus resultados a dois componentes da arquitetura do habitat nestas plantas: o espacial (correspondente ao número e tamanho dos espaços entre os talos) e o estrutural (correspondente ao número, tamanho e largura dos talos). Assim, Amphipoda maiores não estariam limitados pelas larguras e tamanhos dos ramos, mas dependeriam do tamanho dos espaços intersticiais no estabelecimento do menor limite para sua distribuição. Por outro lado, os de pequeno porte não estariam limitados pelo espaço intersticial, mas sim por componentes ligados ao tamanho dos talos da alga. Tais observações foram confirmadas no presente trabalho, onde as menores classes de tamanho de $H$. media foram mais abundantes nas algas mais finamente ramificadas, com talos de menor diâmetro como Gymnogongrus e Pterosiphonia e as maiores classes apresentaram densidades mais elevadas em algas com talos menos ramificados, ou foliáceas, cujos espaços intersticiais são, certamente, maiores como em Sargassum, Pterocladia, Gelidium e Ulva.

Na classificação feita por MORINO (1978 apud LEITE 1996a) para a atividade reprodutiva dos Gammaridea, H. media se enquadra no tipo I, devido à reprodução contínua e registro de fêmeas ovígeras durante todo o ano. Segundo este autor, a 
tendência de possuir um período de vida reduzido e reprodução contínua são características comuns de Amphipoda de regiões de latitudes baixas, o que deve ser o caso das populações de Caiobá.

Segundo SASTRY (1983) nas espécies em que há reprodução contínua, as variações sazonais tornam-se mais evidentes nas espécies de vida longa do que nas de vida curta. As consequências dos distúrbios físicos do meio e predação podem ser melhor observados nas espécies de vida longa, pois elas apresentam um maior período de maturação, necessitando de mais tempo para a superposição de gerações. Nas espécies de vida curta, estes efeitos são rapidamente mascarados, já que muitas gerações são produzidas em curto espaço de tempo. Apesar de atividade reprodutiva um pouco maior no inverno do que em outras estações do ano, as populações de $H$. media de Caiobá podem ser consideradas de vida curta.

O número de ovos encontrados no marsúpio de fêmeas de H. media por LEITE (1996b) variou de 2 a 26. Em Caiobá este número oscilou entre dois e 28, mostrando resultados bastante semelhantes aos registrados para o litoral de São Paulo.

Os comprimentos médios mensais homogêneos, ocorrendo sempre dentro de certos intervalos, obtidos por LEITE (1996b) corroboram o período curto de vida para $H$. media.

VAN DOLAH \& BIRD (1980 apud NELSON 1980) concluíram a partir de dados da literatura que, nos Amphipoda Gammaridea, o número de ovos aumenta com o tamanho da fêmea, o que deve ser uma regra geral, inclusive para $H$. media de Caiobá.

LEITE (1996a) relacionou o número de ovos com o tamanho da cabeça das fêmeas de H. media. A correlação linear encontrada no presente trabalho apresentou um valor do coeficiente linear de Pearson maior que o encontrado pela referida autora. Este fato indica que o comprimento dos quatro primeiros pereonitos é um índice mais preciso que o comprimento da cabeça, na predição do comprimento total do corpo e do número de ovos desta espécie.

SHEADER \& CHIA (1970 apud NELSON 1980) não registraram queda de fecundidade com o aumento da idade e, portanto, com o aumento do tamanho do corpo para Marinogammarus obtusatus (Dahl). Tal afirmação corrobora os presentes dados: fêmeas maiores tendem a apresentar maior número de ovos em seus marsúpios.

Os dados do presente estudo confirmam a necessidade de conservação dos bancos de fitais de Caiobá, pois os mesmos mostraram ser um ecossistema bastante complexo, onde os Amphipoda realizam o seu ciclo de vida. Experimentos de predação em laboratório, provavelmente, virão confirmar que as populações de Amphipoda constituem um importante recurso alimentar para os animais de importância comercial.

AGRADECIMENTOS. À Profa Dra Yoko Wakabara (Instituto Oceanográfico, Universidade de São Paulo) pela confirmação de identificação de Hyale media. Ao Prof. Dr. Ayrton de Mattos (in memorian) e ao M.Sc. José Augusto Cunha, do Departamento de Botânica da Universidade Federal do Paraná pela identificação das algas-substrato do presente estudo. Ao Conselho 
Nacional de Desenvolvimento Científico e Tecnológico (CNPq), na pessoa de seu superintendente, pela concessão de uma bolsa de estudo à primeira autora durante o desenvolvimento do presente trabalho, junto ao Curso de Pós-graduação em Ciências Biológicas-Zoologia da Universidade Federal do Paraná.

\section{REFERÊNCIAS BIBLIOGRÁFICAS}

Bigarella, J.J. 1978. A Serra do Mar e a porção oriental do Estado do Paraná. Curitiba, ADEA, 248p.

BRAWLEY, S. \& X.G. FEY. 1987. Studies of mesoherbivory in aquaria and in an unbarricaded mariculture farm on the chinese coast. Jour. Phycol. 23: 614-623.

DAHL, E. 1948. On the smaller Arthropoda of marine algae, especially in the polyhaline waters off the Swedish West coast. Unters. Oresund 35: 1-193.

DHN. 1986. Tábua das marés. Rio de Janeiro, Diretoria de Hidrografia e Navegação, Marinha do Brasil, 224p.

— 1987. Tábua das marés. Rio de Janeiro, Diretoria de Hidrografia e Navegação, Marinha do Brasil, 224p.

DUBIASKI-SilVA, J. \& S. MASUNARI. 1995. Ecologia populacional dos Amphipoda (Crustacea) dos fitais de Caiobá, Matinhos, Paraná, Brasil. Revta bras. Zool. 12 (2): 373-396.

DuFFY, J.E. 1990. Amphipods on seaweeds: partners or pests? Oecologia 8: 267-276.

DUTRA, R.R.C. 1988. A fauna vágil do fital Pterocladia capillacea (Rhodophyta Gelidiaceae) da Ilha do Mel, Paraná, Brasil. Rev. Brasil. Biol. 48 (3): 589-605. EDGAR, G.J. 1983a. The ecology of South-east Tasmanian phytal animal communities. I. Spatial organization on a local scale. Jour. Exp. Mar. Biol. Ecol. 70: 129-157.

. 1983b. The ecology of South-east Tasmanian phytal animal communities. IV. Factors affecting the distribution of ampithoid amphipods among algae. Jour. Exp. Mar. Biol. Ecol. 70: 205-225.

GiBBons, M.J. 1988. The impact of wave exposure on the meiofauna of Gelidium pristoides (Turner) Kuetzing (Gelidiales: Rhodophyta). Estuar. Coast. Shelf Scien. 27: 581-593.

HACKER, S.D. \& R.S. STENECK. 1990. Habitat architecture and body-size-dependent habitat selection of a phytal amphipod. Ecology 71 (6): 2269-2285.

JAKOBI, H. 1954. Espécies novas de Harpacticoidea (Copepoda Crustacea) encontradas nas algas marinhas do litoral do Paraná e Santa Catarina. Bol. Inst. Oceanogr. 5 (1-2): 189-212.

1962. Harpacticoidea e Syncarida troglobiontes (Crustacea). Bol. Univer. Paraná 21: 1-19.

Joly, A.B. 1951. Contribuição para o conhecimento da flora algológica marinha do Estado do Paraná. Bol. Inst. Oceanogr. 2 (1): 125-138.

KIKUCHI, T. \& J.M. PÉRĖs. 1977. Consumer ecology of seagrass beds, p. 147-193. In: C.P. MC RoY \& C. HelfFERICH (Eds). Seagrass Ecossystem. New York, M Dekker, IX+314p. 
LANCEllotti, D.A. \& R.G. TRUCCO. 1993. Distribution patterns and coexistence of six species of the amphipod genus Hyale. Mar. Ecol. Progr. Ser. 93: 131-141.

LEITE, F.P.P. 1996a. Crescimento e reprodução de Hyale media Dana (Amphipoda, Gammaridea, Hyalidae) associada à Sargassum cymosum C. Agardh. Revta bras. Zool. 13 (3): 597-606.

1996b. Fecundidade de sete espécies de gamarídeos (Crustacea, Amphipoda, Gammaridea) associados à alga Sargassum cymosum. Iheringia, Sér. Zool., (80): 39-45.

Leite, F.P.P.; Y. WAKabara \& A.S. TARARAM. 1986. On the morphological variations in ooostegites of gammaridean species (Amphipoda). Crustaceana $51(1): 77-94$.

LEWIS, F.G. III. 1987. Crustacean epifauna of seagrass and macroalga in Apalachee Bay, Florida, USA. Mar. Biol. 94 (2): 219-229.

Loyola E Silva, J. 1960. Sphaeromatidae do litoral brasileiro. Bol. Univers. Paraná 4: 1-182.

LUCZKOVICH, J.J. 1988. The role of prey detection in the selection of prey by pinfish Lagodon rhomboides (Linnaeus). Jour. Exp. Mar. Biol. Ecol. 123: 15-30.

MAACK, R. 1968. Geografia física do Estado do Paraná. Rio de Janeiro, J. Olimpio, $2^{\mathrm{a}}$ ed., $450 \mathrm{p}$.

Masunari, S. 1986. Copepoda Harpacticoidea dos fitais do litoral do Paraná. Ciênc. Cult. 38 (7): 1010.

1987. Ecologia das comunidades fitais. In: I Simpósio sobre

Ecossistemas da Costa Sul e Sudeste Brasileira: síntese dos conhecimentos. Publicações ACIESP nº 54-1: 195-233.

MASUNARI, S. \& L. ForNeris. 1981. O ecossistema fital - uma revisão. In: Seminários de Biologia Marinha. Rio de Janeiro, Academia Brasileira de Ciências, p.149-172.

NELSON, W.G. 1980. Reproductive patterns of gammaridean amphipods. Sarsia 65: $61-71$.

REMANE, A. 1933. Verteilung und Organisation der benthonishen Mikrofauna der Kieler Bucht. Wiss. Meeresunters., Kiel, 21: 161-221.

RYER, C.H. 1988. Pipefish foraging: effects of fish size, prey size and altered habitat complexity. Mar. Ecol. Progr. Ser. 48 (1): 37-45.

RyER, C.H. \& R.J. ORTH. 1987. Feeding ecology of the Northern pipefish, Syngnathus fuscus, in a seagrass community of the lower Cheasapeake Bay. Estuaries 10 (4): 330-336.

SAstry, A.N. 1983. Ecological aspects of reproduction. In: D.E. Bliss (Ed.). The biology of Crustacea. Vernberg, F.J. \& W.b. Vernberg (Eds). Environmental adaptations: 179-270. New York, Academic, Vol. 8, XXVI+383p.

SCHNEIDER, F.I. \& K.H. MANN. 1991a. Species specific relationships of invertebrates to vegetation in a seagrass bed. I. Correlational studies. Jour. Exp. Mar. Biol. Ecol. 145: 101-117.

. 1991b. Species specific relationships of invertebrate to vegetation in a seagrass bed. II. Experiments on the importance of macrophyte shape, epiphyte 
cover and predation. Jour. Exp. Mar. Biol. Ecol. 145: 119-139.

TARARAM, A. S.; Y. WaKabara \& F.P.D. LeIte. 1985. Feeding habits of Hyale media (Dana, 1953) (Crustacea-Amphipoda). Bol. Inst. Oceanogr. USP 33 (2): 193-199.

- 1986. Vertical distribution of amphipods living on algae of a Brazilian intertidal rocky shore. Crustaceana 51 (2): 83-187.

Tararam, A.S.; H.S.L. Mesquita; Y. WaKabara \& C.A Peres. 1990. Food ingestion and assimilation by Hyale media (Dana, 1853) (Crustacea -Amphipoda). Bol. Inst. Oceanogr. USP 38 (1): 11-21.

UnderWOOD, A.J. \& P.H. VERTEGEN. 1988. Experiments on the association between the intertidal amphipod Hyale media Dana and the limpet Cellana tramoserica (Sowerby). Jour. Exp. Mar. Biol. Ecol. 119: 83-98.

VIEJO, R.M. \& J. ARRONTES. 1992. Interactions between mesograzers inhabiting Fucus vesiculosus in Northern Spain. Jour. Exp. Mar. Biol. Ecol. 162: 97-111. WAKABARA, Y.; A. S. TARARAM \& A. M. TAKEDA.1983. Comparative study of the amphipod fauna living on Sargassum of two Itanhaém shores, Brazil. Jour. Crust. Biol. 3 (4): 602-607.

WIESER, W. 1951. Ueber die quantitative Bestimmung der Algenbewohnenden Mikrofauna felsiger Meerekuesten. Oikos 3 (1): 124-131.

Recebido em 26.IX.1996; aceito em 05.XI.1997. 